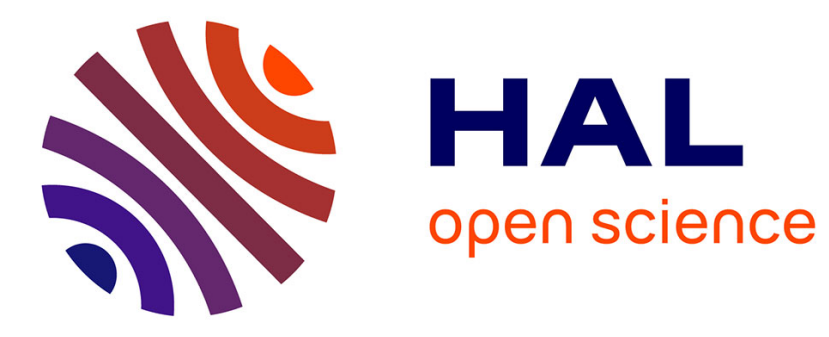

\title{
Fluid structure interaction problems in large deformation
}

Patrick Le Tallec, Jean-Frédéric Gerbeau, Patrice Hauret, Marina Vidrascu

\section{To cite this version:}

Patrick Le Tallec, Jean-Frédéric Gerbeau, Patrice Hauret, Marina Vidrascu. Fluid structure interaction problems in large deformation. Comptes Rendus Mécanique, 2005, 333, pp.910-922. hal00111445

\section{HAL Id: hal-00111445 \\ https://hal.science/hal-00111445}

Submitted on 23 Dec 2017

HAL is a multi-disciplinary open access archive for the deposit and dissemination of scientific research documents, whether they are published or not. The documents may come from teaching and research institutions in France or abroad, or from public or private research centers.
L'archive ouverte pluridisciplinaire HAL, est destinée au dépôt et à la diffusion de documents scientifiques de niveau recherche, publiés ou non, émanant des établissements d'enseignement et de recherche français ou étrangers, des laboratoires publics ou privés. 


\title{
Fluid Structure Interaction Problems in Large Deformation
}

\section{Simulation numérique des problèmes d'interaction fluide structure en grandes déformations}

\author{
Patrick Le Tallec ${ }^{\mathrm{a}}$ Jean-Frédéric Gerbeau ${ }^{\mathrm{b}}$ Patrice Hauret ${ }^{\mathrm{c}}$ Marina Vidrascu $^{\mathrm{d}}$ \\ ${ }^{a}$ Ecole Polytechnique, F 91128 Palaiseau Cedex \\ ${ }^{\mathrm{b}}$ INRIA Rocquencourt, B.P. 105, F 78153 Le Chesnay Cedex \\ ${ }^{\mathrm{c}}$ Graduate Aeronautical Labs, California Institute of Technology, 1200 E California Blvd, Pasadena, CA 91 125, USA \\ ${ }^{\mathrm{d}}$ INRIA Rocquencourt, B.P. 105, F 78153 Le Chesnay Cedex
}

\begin{abstract}
The present paper deals with the simulation of fluid structure interaction problems in large deformation, and discusses two aspects of their numercial solution: - the derivation of enery conserving time integration schemes in presence of fluid structure coupling, moving grids, and nonlinear kinematic constraints such as incompressibility and contact, - the introduction of adequate preconditioners efficiently chaining local fluid and stucture solvers. Solutions are proposed, analyzed and tested using nonlinear energy correcting terms, and added mass based Dirichlet Neumann preconditioners. Numerical applications include nonlinear impact problems in elastodynamics and blood flows predictions within flexible arteries.
\end{abstract}

\section{Résumé}

Du fait des fortes nonlinéarités du problème posé, la simulation de phénomènes d'interaction fluide structure en grands déplacements et vitesses modérées conduit à plusieurs difficultés numériques : respect numérique des mécanismes de conservation d'énergie dans le traitement des grilles mobiles, des forces de raideur, de la synchronisation des forces de contact et d'interface d'une part, constructrion de préconditionneurs adaptés permettant l'utilisation efficace d'algorithmes de couplage résolvant de manière successive et découplée les parties fluide et structure, d'autre part.

L'article introduit d'abord la formulation mathématique du problème de couplage de fluide structure en grands déplacements dans un cadre de biomécanique. Il explique l'impact des diverses nonlinéarités mécaniques et cinématiques du problème sur les schémas d'intégration numérique en temps, et propose une stratégie systématique de corrections nonlinéaires permettant de restaurer les propriétés fondamentales de conservation d'énergie après discrétisation. Cette stratégie, proposée initialement dans [7] et fondée sur un remplacement de dérivées d'énergie par des différences divisées, est appliquée à toutes les composantes du problème : contraintes élastiques, incompressibilité, contact, termes de convection dans le fluide. L'article rappelle ensuite les difficultés de convergence qui peuvent se produire dans le traitement itératif de ces problèmes, et explique l'intérêt de préconditionneurs de type masse ajoutée dans une approche multidomaine (Dirichlet Neumann) de la résolution numérique. Ces différents aspects sont illustrés sur des applications numériques tridimensionnelles, d'une part sur des problèmes 


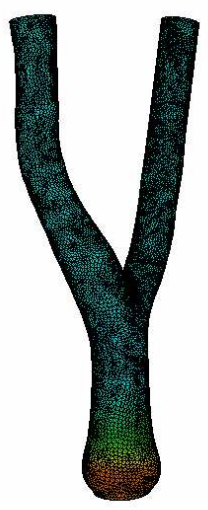

FIG. 1. A pressure wave inside an aortic bifurcation. Onde de pression à l'intérieur d'une bifurcation aortique.

d'élastodynamique nonlinéaire étudiant le comportement en temps long d'une structure hyperélastique incompressible, d'autre part sur des problèmes d'hémodynamique étudiant les écoulements sanguins dans des anévrismes ou dans des artères souples.

Keywords : nonlinear elastodynamics, time integration, energy conservation, fluid structure interaction, added mass, preconditioner.

Mots clés : élastodynamique nonlinéaire, intégration en temps, conservation de l'énergie, interaction fluide structure, masse ajoutée, préconditionneur.

\section{Introduction}

The recent interest in biomechanical problems has introduced new types of fluid structure interaction problems where a complex flexible structure such as artery walls or cardiac muscles interacts with the flow of an incompressible fluid, namely the blood. This has motivated a renewed interest in the development and analysis of efficient (accurate) numerical tools in nonlinear dynamics, in kinematic coupling, and in domain decomposition algorithms in order to properly handle issues such as discrete conservation of energy, time preservation of (nonlinear) kinematic constraints (incompressibility, ...), and numerical efficiency.

Indeed, because of large deformation, contact, or of kinematic constraints such as incompressibility, these systems have a highly nonlinear behavior, which affects the global conservation properties of most linear schemes $[11,16]$. This is rather disturbing in fluid interaction problems because existence, convergence and stability results are all based on energy estimates [4,5]. Therefore, one needs to introduce energy correction terms in the numerical approximation of the original problem. For pure elastodynamics problems, this has been done in [14] for quadratic energy in large displacements, with a second correction added by [7] to handle more general situations. Herein, using the ideas already introduced in [15] and in [8], we will extend the strategy of [7] for structural problems in presence of contact and of internal flows

Email addresses: Patrick Le Tallec@polytechnique.fr (Patrick Le Tallec), Jean-Frédéric.Gerbeau@inria.fr (Jean-Frédéric Gerbeau), phauret@aero.caltech.edu (Patrice Hauret), Marina.Vidrascu@inria.fr (Marina Vidrascu). 


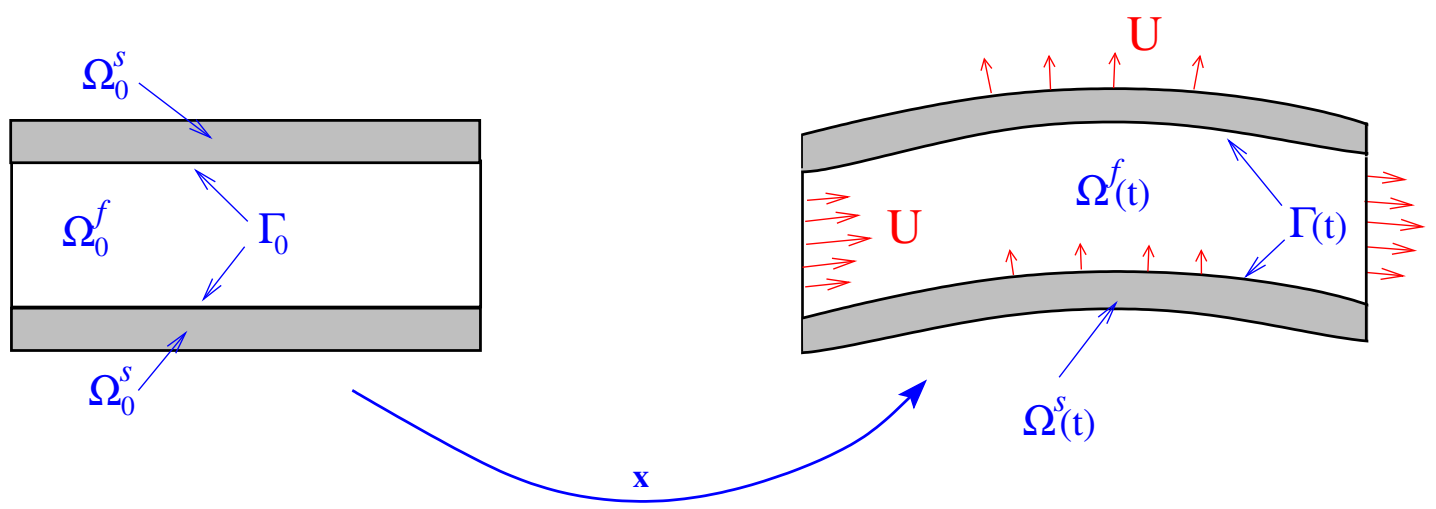

FIG. 2. Configuration of the fluid structure problem. Configuration du problème posé.

discretized on moving grids. The key at this level is to derive specific energy correction terms to handle the coupling between domain transport, fluid grid motion and time derivatives of contact forces.

Because of the large size of the resulting problems, efficient solvers must also be developed, respecting the basic coupling mechanisms between the different subdomains, while retaining the formulation and specific complexity of the local solvers available for the separate solution of the structural problem on one hand, and on the incompressible flow problem on the other hand. The Dirichlet Neumann strategy originally introduced and analyzed by [12] for elliptic problems and by [17] for fluid structure problems can be a reasonable candidate, but lacks efficiency in very large scale systems. As observed in [6], efficiency is restored when respecting the added mass effects in this Dirichlet Neumann algorithm.

The purpose of the present paper is then to describe, explain and justify on a significant model problem $(\S 2)$, the mechanism, role and importance of energy corrections (§3), and to explain the philosophy of added mass preconditioners for Dirichlet Neumann algorithms $(\S 4)$.

\section{The mechanical problem}

\subsection{The system of incompressible elastodynamics in large deformation}

The system under study occupies a moving domain $\Omega(t)$ in its present configuration. It is made of a fluid in motion in a deformable part $\Omega^{f}(t)$ of $\Omega(t)$ and of a deformable flexible structure which lies on the complement $\Omega^{s}(t)$ of $\Omega^{f}(t)$ in $\Omega(t)$ (Figure 2). The problem consists in finding both the time evolution of this configuration, and the velocity $U:=\frac{d \mathbf{x}}{d t}$ and Cauchy stress tensor $\sigma$ within the fluid and the structure.

The time evolution, and the associated stress distribution within the structure is best described in a known reference configuration $\Omega_{0}^{s}$ where both the equation of motion and the constitutive law are easy to write and to identify. The evolution of the structure is then governed by an initial boundary value problem set on $\Omega_{0}^{s}$ whose main unknown is the position $\mathbf{x}(X, t)$ of the different material points $X$ at time $t$ :

$$
\begin{aligned}
& m(\ddot{\mathbf{x}}, \hat{U})+a(\nabla \mathbf{x}, \nabla \hat{U})=\int_{\Omega} f \cdot \hat{U}+\int_{\partial \Omega} g \cdot \hat{U}+\int_{\partial \Omega_{c}} \lambda \nu \cdot \hat{U}, \forall \hat{U} \in \mathcal{U}, \\
& \int_{\Omega}\left(\operatorname{det}\left(C^{1 / 2}(\nabla \mathbf{x})\right)-1+\epsilon p\right) \hat{p}=0, \forall \hat{p} \in \mathcal{P}, \\
& \mathbf{x} \cdot \nu \geq g_{0} \text { and } \lambda\left(\mathbf{x} \cdot \nu-g_{0}\right)=0 \text { on } \partial \Omega_{c} .
\end{aligned}
$$


Above, the structural mass operator $m$ has the usual linear expression encountered in Lagrangian dynamics

$$
m(\ddot{\mathbf{x}}, \hat{U})=\int_{\Omega_{0}^{s}} \rho \ddot{\mathbf{x}} \cdot \hat{U} d x .
$$

When dealing with incompressible or almost incompressible elastic materials in large deformation, the stiffness term $a(\nabla \mathbf{x}, \nabla \hat{U})$ is best defined in mixed form as $a(\nabla \mathbf{x}, \nabla \hat{U})=\int_{\Omega} F \cdot \Sigma(\nabla \mathbf{x}) \cdot \nabla \hat{U}$ with $\Sigma$ the second Piola-Kirchhoff stress tensor given by :

$$
\Sigma=2 \frac{\partial \mathcal{W}}{\partial C}-2 p \frac{\partial \operatorname{det} C^{1 / 2}}{\partial C},
$$

where $p$ denotes the hydrostatic pressure, $\mathcal{W}$ the stored elastic energy, which is a given function of the right Cauchy-Green strain tensor $C=F^{t} \cdot F$, and $F=\nabla \mathbf{x}$ denotes the deformation gradient.

The above formulation involve three major nonlinear effects :

- a transport term $F=\nabla \mathbf{x}$ in factor of the stress tensor $\Sigma$, due to the pull back of the equation of motion from the present configuration to the reference one,

- a nonlinear incompressibility constraint on det $\left(C^{1 / 2}(\nabla \mathbf{x})\right)-1$ written in terms of the Cauchy strain tensor $C(\nabla \mathbf{x})$ for a simpler verification of energy conservation,

- a frictionless contact constraint $\mathbf{x} \cdot \nu \geq 0$ imposed on a part $\partial \Omega_{c}$ of its boundary where the displacement $\mathbf{x} \cdot \nu$ normal to a given obstacle cannot exceed a given threshold $g_{0}$. In practice, this frictionless contact constraint is often handled by a penalty approach giving the normal reaction $\lambda$ as a function of the interpenetration distance $\left|\mathbf{x} \cdot \nu-g_{0}\right|_{-}=\max \left(0, g_{0}-\mathbf{x} \cdot \nu\right)$ by $\lambda=\frac{1}{\epsilon_{c}}\left|\mathbf{x} \cdot \nu-g_{0}\right|_{-}$. where $\epsilon_{c}$ is a small penalty coefficient.

Moreover, $\epsilon \geq 0$ is also a small parameter, whose inverse can be interpreted as the bulk modulus. The formulation inroduced in (1-3) handles quasi incompressible as well as truly incompressible materials and leads to finite element formulations which converge uniformly with respect to the bulk modulus.

\subsection{Fluid structure interactions in large deformation}

In fluid structure interaction problems, in order to evaluate the strain field or write the elastic constitutive laws inside the structure, it is again very convenient to transport the conservation laws for both the fluid and the structure on a fixed reference configuration $\Omega_{0}$. The choice of the configuration $\Omega_{0}$ and of the map $\mathbf{x}: \Omega_{0} \rightarrow \Omega(t)$ (and hence of its Jacobian $J=\operatorname{det} \frac{\partial \mathbf{x}}{\partial \mathbf{x}_{0}}$ and of the underlying grid velocity $U_{G}=\left(\frac{\partial \mathbf{x}}{\partial t}\right)_{\mid \mathbf{x}_{0}}$ ) may be arbitrary (Arbitrary Lagrangian Eulerian (ALE) formulation), but, as seen above, on the structure $\Omega^{s}$, the equations are much simpler when the point $\mathbf{x}\left(\mathbf{x}_{0}, t\right)$ corresponds to the present position $\mathbf{x}^{s}\left(\mathbf{x}_{0}, t\right)$ of the material point which was located in $\mathbf{x}_{0}$ at time $t_{0}$. The mapping $\mathbf{x}^{f}$ from $\Omega_{0}^{f}$ onto $\Omega^{f}(t)$ defining the present position of each discretization grid point inside the fluid is then a user defined extension $\mathbf{x}^{f}=\operatorname{Ext}\left(\mathbf{x}_{\mid \Gamma_{0}}^{s}\right)$ of the structural deformation, matching this deformation on the fluid structure interface.

The structure is again supposed to be nonlinear incompressible elastic, and interacts with a viscous incompressible fluid of given density $\rho$ which perfectly sticks to its boundary, meaning that the fluid particles must follow the structure during the motion. In this framework, using the nonconservative formulation usually employed when dealing with incompressible fluids, the mechanical evolution of the global fluid structure system is governed by the following equations :

Find the structural deformation $\mathbf{x}^{s} \in V^{s}$, the pressure $p \in Q=L^{2}\left(\Omega_{0}\right)$ in the fluid and in the solid, the fluid velocity $U^{f} \in V^{f}$, the interface traction $g_{\Gamma} \in W_{\Gamma}=\left(H^{1 / 2}\left(\Omega_{0}\right)\right)^{\prime}$, the contact force $\lambda$ on $\partial \Omega_{c}$, and the fluid configuration mapping $\mathbf{x}^{f} \in V^{f}$ such that 


$$
\begin{aligned}
& \int_{\mathbf{x}^{f}\left(\Omega_{0}^{f}, t\right)} \operatorname{div}\left(\rho U^{f}\right) \hat{q}+\int_{\Omega_{0}^{s}}\left(\operatorname{det}\left(C^{1 / 2}(\nabla \mathbf{x})\right)-1+\epsilon p\right) \hat{q}=0, \\
& \forall \hat{q}: \Omega_{0} \rightarrow \mathbb{R}, \quad \text { (mass and volume conservation) } \\
& m^{s}\left(\ddot{\mathbf{x}}^{s}, \hat{U}^{s}\right)+\int_{\mathbf{x}^{f}\left(\Omega_{0}^{f}, t\right)} \rho\left({\frac{\partial U^{f}}{\partial t}}_{\mathbf{x}_{0}}+\left(U^{f}-U^{G}\right) \cdot \nabla U^{f}\right) \cdot \hat{U}^{f} \\
& +a^{s}\left(\nabla \mathbf{x}^{s}, \nabla \hat{U}^{s}\right)+\int_{\mathbf{x}^{f}\left(\Omega_{0}^{f}, t\right)}\left(\mu\left(\nabla_{x} U^{f}+\nabla_{x}^{t} U^{f}\right)-p I d\right): \frac{\partial \hat{U}^{f}}{\partial x} \\
& =\int_{\Omega(t)} f \cdot \hat{U}+\int_{\partial \Omega(t)} g \cdot \hat{U}+\int_{\partial \Omega_{c}} \lambda \nu \cdot \hat{U}+\int_{\Gamma_{0}} g_{\Gamma} \cdot\left(\operatorname{tr}\left(\hat{U}^{s}\right)_{\mid \Gamma}-\operatorname{tr}\left(\hat{U}^{f}\right)_{\mid \Gamma}\right), \\
& \forall\left(\hat{U}^{s}, \hat{U}^{f}\right) \in V^{s} \times V^{f}, \quad \text { (momentum conservation) } \\
& \operatorname{tr}\left(\mathbf{x}^{s}\right)_{\mid \Gamma}=\operatorname{tr}\left(\mathbf{x}_{0}\right)_{\mid \Gamma}+\int_{0}^{t} \operatorname{tr}\left(U^{f}\right)_{\mid \Gamma}(\tau) d \tau, \quad \text { (kinematic continuity) } \\
& \mathbf{x}^{f}=\operatorname{Ext}\left(x_{\mid \Gamma_{0}}^{s}\right) . \quad \text { (fluid configuration map) }
\end{aligned}
$$

The presence of the fluid brings in a new nonlinear convection term $\rho\left(U^{f}-U^{G}\right) \cdot \nabla U^{f}$. Observe in addition that the kinematic continuity condition imposed at the interface between the fluid and the structure is expressed in displacements. Indeed, the structural velocity is only in $L^{2}\left(\Omega^{s}\right)$, and therefore we cannot define its trace on the interface.

\section{Energy conserving implicit schemes}

\subsection{Basic time integration schemes}

A standard implicit scheme in elastodynamics uses a trapezoidal rule for time integration combined with stress averaging [2]. For nonlinear problems, Simo or Crisfield [14,3] have proposed to use in addition a transport averaging, which means that each integrand $(\cdot)_{n+1 / 2}$ is predicted as follows

$$
\begin{aligned}
& (F \cdot \Sigma(\nabla \mathbf{x}))_{n+1 / 2}=\frac{1}{2}\left(\nabla \mathbf{x}_{n+1}+\nabla \mathbf{x}_{n}\right) \cdot \Sigma_{n+1 / 2} \text { (transport averaging) } \\
& \Sigma_{n+1 / 2}=\left(\mathcal{W}_{, C}\left(C\left(\nabla \mathbf{x}_{n+1}\right)\right)+\mathcal{W}_{, C}\left(C\left(\nabla \mathbf{x}_{n}\right)\right)\right) \\
& \quad-p_{n+1} \frac{\partial \operatorname{det}\left(C^{1 / 2}\left(\nabla \mathbf{x}_{n+1}\right)\right)}{\partial C}-p_{n} \frac{\partial \operatorname{det}\left(C^{1 / 2}\left(\nabla \mathbf{x}_{n}\right)\right)}{\partial C} \text { (stress averaging) } \\
& \int_{\Omega}\left(\operatorname{det}\left(C^{1 / 2}\left(\nabla \mathbf{x}_{n+1}\right)\right)-1+\epsilon p_{n+1}\right) \hat{p}=0, \forall \hat{p},\left(\text { incompressibility at time } t_{n+1}\right), \\
& U_{n+1 / 2}=\frac{\mathbf{x}_{n+1}-\mathbf{x}_{n}}{\Delta t_{n}}=\frac{1}{2}\left(U_{n+1}+U_{n}\right),(\text { velocity construction) } \\
& (\ddot{\mathbf{x}})_{n+1 / 2}=\frac{U_{n+1}-U_{n}}{\Delta t_{n}}(\text { acceleration }) .
\end{aligned}
$$

In theory, the above time integration schemes have good properties with respect to energy conservation, achieving second order accurate conservation, with an error vanishing at the linear limit. For example, a mid point integration of the mechanical work developed by the elastic stress yields 


$$
\begin{aligned}
& \frac{F_{n}+F_{n+1}}{2} \cdot\left(\mathcal{W}_{,_{C}}\left(C_{n+1}\right)+\mathcal{W}_{, C}\left(C_{n}\right)\right) \cdot \nabla U_{n+1 / 2} \\
& =\left(\mathcal{W}_{, C}\left(C_{n+1}\right)+\mathcal{W}_{, C}\left(C_{n}\right)\right) \cdot \frac{C_{n+1}-C_{n}}{2 \Delta t_{n}} \\
& =\frac{1}{\Delta t_{n}}\left[\mathcal{W}\left(C_{n+1}\right)-\mathcal{W}\left(C_{n}\right)+C \frac{\partial^{3} \mathcal{W}}{\partial C^{3}}\left(C_{*}\right) \cdot\left(C_{n+1}-C_{n}\right)^{3}\right] \\
& =\frac{1}{\Delta t_{n}}\left[\mathcal{W}\left(C_{n+1}\right)-\mathcal{W}\left(C_{n}\right)\right]+c \Delta t_{n}^{2}
\end{aligned}
$$

with a similar behavior for the incompressibility terms $p \frac{\partial \operatorname{det} C^{1 / 2}}{\partial C}$.

In practice, as observed on Figure 3 for both the trapezoidal and the mid point schemes, such a second order conservation is not good enough for nonlinear structures, and numerical instabilities are often observed in real life simulations.

\subsection{Energy Corrections on the structure}

Nonlinear corrections are then needed, with different choices proposed in the litterature. We have tested and adopted a nonlinear and non symmetric correction term proposed by Gonzalez [7], where the elastic

stress average $\Sigma_{n+1 / 2}^{c}=\left(\mathcal{W}_{, C}\left(C_{n+1}\right)+\mathcal{W}_{,_{C}}\left(C_{n}\right)\right)$ acting on the Cauchy strain variation is replaced by the following divided difference :

$$
\begin{aligned}
& \Sigma_{n+1 / 2}^{c}=2 \frac{\partial \mathcal{W}}{\partial C}\left(C_{n+1 / 2}\right) \\
& +2\left(\mathcal{W}\left(C_{n+1}\right)-\mathcal{W}\left(C_{n}\right)-\frac{\partial \mathcal{W}}{\partial C}\left(C_{n+1 / 2}\right): \delta C_{n+1 / 2}\right) \frac{\delta C_{n+1 / 2}}{\delta C_{n+1 / 2}: \delta C_{n+1 / 2}}
\end{aligned}
$$

with $\delta C_{n+1 / 2}=C_{n+1}-C_{n}$. It was rapidly observed in [8] that a similar correction must be added to the pressure term, yielding

$$
\begin{aligned}
& \Sigma_{n+1 / 2}^{i n c}=-\left(p_{n+1}+p_{n}\right)\left[\frac{\partial \operatorname{det} C^{1 / 2}}{\partial C}\left(C_{n+1 / 2}\right)+\right. \\
& \left.\left(\operatorname{det} C_{n+1}^{1 / 2}-\operatorname{det} C_{n}^{1 / 2}-\frac{\partial \operatorname{det} C^{1 / 2}}{\partial C}\left(C_{n+1 / 2}\right): \delta C_{n+1 / 2}\right) \frac{\delta C_{n+1 / 2}}{\delta C_{n+1 / 2}: \delta C_{n+1 / 2}}\right] .
\end{aligned}
$$

By construction and from the incompressibility constraint satisfied at times $t_{n}$ and $t_{n+1}$, we then directly have

$$
\frac{1}{2} \Sigma_{n+1 / 2}: \delta C_{n+1 / 2}=\frac{1}{2}\left(\Sigma_{n+1 / 2}^{c}+\Sigma_{n+1 / 2}^{i n c}\right): \delta C_{n+1 / 2}=\mathcal{W}\left(C_{n+1}\right)-\mathcal{W}\left(C_{n}\right),
$$

implying exact energy conservation, even at the incompressible limit. The resulting numerical tests observed on a simple incompressible beam are then quite convincing (fig. 3) and in sharp contrast with the diverging results of the original trapezoidal rule.

But, even after these first two corrections, the proposed scheme does not handle well contact conditions. In the framework of frictionless contact, both Laursen and Chawla [9] and Armero and Petcz [1] observing such difficulites, have shown the interest of the persistency condition $\lambda(t, x) \frac{d}{d t}\left(\mathbf{x} \cdot \nu-g_{0}\right)=0$ to obtain energy conservation in the discrete framework. Nevertheless, as underlined in [10], both contributions encounter a difficulty in enforcing standard Kuhn-Tucker conditions associated to frictionless contact. 


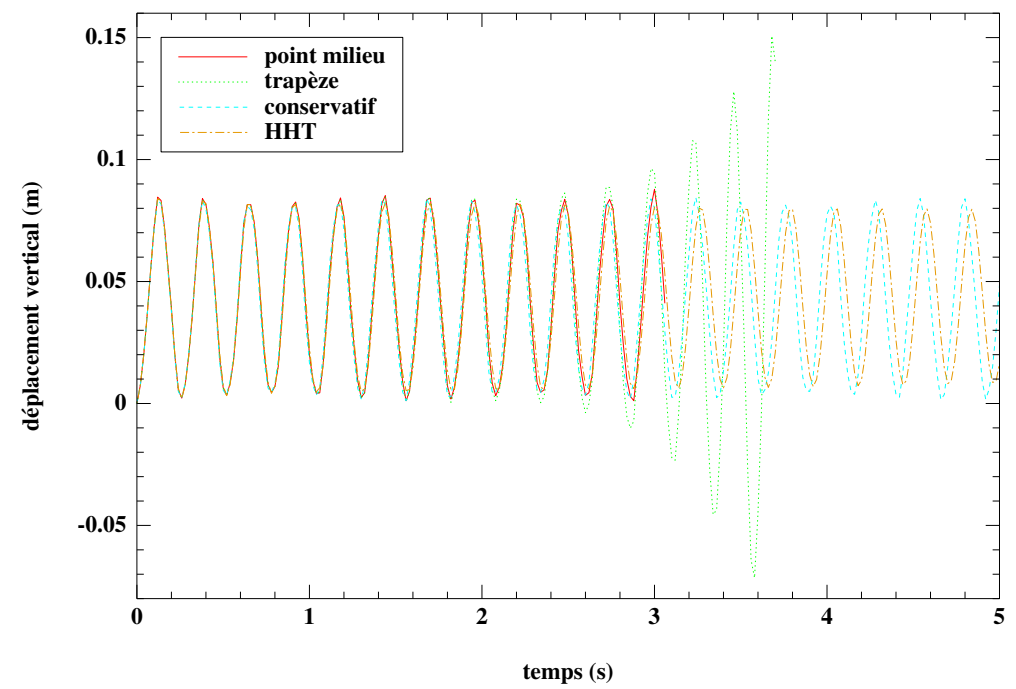

FIG. 3. Long term energy evolution of an oscillating nonlinear beam with different numerical schemes. Evolution en temps long de l'énergie dans une poutre oscillante pour différents schémas.

This difficulty is resolved in [10], by introducing a discrete jump in velocities during impact, making possible the enforcement of contact conditions at each time step, at the computational price of resolving a problem on the jump in velocities. In the framework of the present penalized enforcement of the contact condition (3), the energy correction (9) can be adapted to enforce the standard Kuhn-Tucker contact conditions at entire time steps [8]. The trick is to treat the contact constraint exactly as the incompressiblity constraint averaging separately the geometric update (transport) of the normal $\nu$ and the kinetic force $\lambda$, while replacing local derivatives by divided differences.

To reproduce in the discrete framework the previous conservation properties, we propose the following midtime approximation of the normal vector

$$
\nu_{n+1 / 2}=\nu\left(\mathbf{x}_{n+1 / 2}\right)+\left[\mathbf{x}_{n+1} \cdot \nu\left(\mathbf{x}_{n+1}\right)-\mathbf{x}_{n} \cdot \nu\left(\mathbf{x}_{n}\right)-\nu\left(\mathbf{x}_{n+1 / 2}\right) \cdot \delta \mathbf{x}\right] \frac{\delta \mathbf{x}}{\delta \mathbf{x} \cdot \delta \mathbf{x}},
$$

where $\nu\left(\mathbf{x}_{n+1 / 2}\right)$ is the normal outward unit vector to the obstacle at mid point $\mathbf{x}_{n+1 / 2}$ and $\delta \mathbf{x}=$ $\mathbf{x}_{n+1}-\mathbf{x}_{n}$ is the displacement update between two successive time steps. Observe that we always have by construction

$$
\nu_{n+1 / 2} \cdot \delta \mathbf{x}=\mathbf{x}_{n+1} \cdot \nu\left(\mathbf{x}_{n+1}\right)-\mathbf{x}_{n} \cdot \nu\left(\mathbf{x}_{n}\right):=\delta g
$$

and that for a plane obstacle for which $\nu\left(\mathbf{x}_{n+1 / 2}\right)=\nu\left(\mathbf{x}_{n+1}\right)=\nu\left(\mathbf{x}_{n}\right)=\nu$, the above construction simply reduces to $\nu_{n+1 / 2}=\nu$. Similarly, we propose the following update of the reaction force

$$
\lambda_{n+1 / 2}=\frac{1}{\epsilon_{c} \delta g}\left(\frac{1}{2}\left|\mathbf{x}_{n+1} \cdot \nu_{n+1}-g_{0}\right|_{-}^{2}-\frac{1}{2}\left|\mathbf{x}_{n} \cdot \nu_{n}-g_{0}\right|_{-}^{2}\right),
$$

so that we have by construction

$$
\int \lambda_{n+1 / 2} \nu_{n+1 / 2} \cdot \delta \mathbf{x}=\int \frac{1}{2 \epsilon_{c}}\left|\mathbf{x}_{n+1} \cdot \nu_{n+1}-g_{0}\right|_{-}^{2}-\frac{1}{2 \epsilon_{c}}\left|\mathbf{x}_{n} \cdot \nu_{n}-g_{0}\right|_{-}^{2},
$$

that is perfect conservation of the penalty energy.

To validate the proposed energy conserving impact formulation, let us consider an elastic ball presenting a small cylindrical hole around one of its diameters (Figure 5).

Four snapshots of the impact simulation are shown on figure 5. As illustrated on figure 6 , the evolution 


\begin{tabular}{|l|l|}
\hline radius & $0.1 \mathrm{~m}$ \\
density & $1200 \mathrm{~kg} / \mathrm{m}^{3}$ \\
Young's modulus & $0.2 \mathrm{M} \mathrm{Pa}$ \\
Poisson's ratio & 0.33 \\
initial distance of the center & \\
of the ball to the wall & $0.12 \mathrm{~m}$ \\
initial velocity & $0.4 \mathrm{~m} / \mathrm{s}$ \\
$\epsilon_{c}$ & $1 . \mathrm{E}-4$ \\
time step & $0.002 \mathrm{~s}$ \\
T & $1.0 \mathrm{~s}$ \\
$\#$ nodes in the mesh & 11.160 \\
\hline
\end{tabular}

FIG. 4. Data for the constitutive Saint-Venant Kirchhoff material and for the geometry of the ball. Données constitutives et géométriques pour la balle élastique.

of discrete energy in the ball during the dynamics is very sensitive to the time integration strategy.

In particular, the discrete energy explodes when using a midpoint scheme or a trapezoidal scheme. The conservative Gonzalez scheme enriched with our energy conserving impact formulation keeps its promise and the relative loss of energy through the impact is $1.8 \mathrm{E}-4$, only depending on the accuracy of the Newton's solver.

\subsection{Energy conserving scheme for the fluid structure problem}

We now complete the above nonlinear energy conserving scheme used on the structure by a similar scheme integrating the fluid equation at time $n+1 / 2$ by a second order Crank Nicholson scheme with

$$
\left(\frac{\partial U^{f}}{\partial t}\right)_{n+1 / 2}=\frac{U_{n+1}^{f}-U_{n}^{f}}{\Delta t}, \quad U_{n+1 / 2}^{G}=\frac{\mathbf{x}_{n+1}^{f}-\mathbf{x}_{n}^{f}}{\Delta t}
$$

while averaging all expressions at time $t_{n+1 / 2}$ by

$$
(\cdot)_{n+1 / 2}=\frac{(\cdot)_{n+1}+(\cdot)_{n}}{2} .
$$

With this choice, the time discrete problem is : At each time $t_{n+1}$, find the structural deformation $\mathbf{x}_{n+1}^{s} \in V^{s}$, the pressure $p_{n+1 / 2} \in Q$, the fluid velocity $U_{n+1}^{f}$, the interface traction $\left(g_{\Gamma}\right)_{n+1} \in W_{\Gamma}$, the contact force $\lambda_{n+1 / 2}$ and the fluid configuration mapping $\mathbf{x}_{n+1}^{f} \in V^{f}$ such that

$$
\int_{x^{f}\left(\Omega_{0}^{f}, t_{n+1 / 2}\right)} \operatorname{div}\left(U_{n+1 / 2}^{f}\right) \hat{q}+\int_{\Omega_{0}^{s}}\left(\operatorname{det}\left(C^{1 / 2}\left(\nabla \mathbf{x}_{n+1}\right)\right)-1+\epsilon p_{n+1}\right) \hat{q}=0,
$$

$$
\forall \hat{q}: \Omega_{0} \rightarrow \mathbb{R},
$$

for mass and volume conservation, 

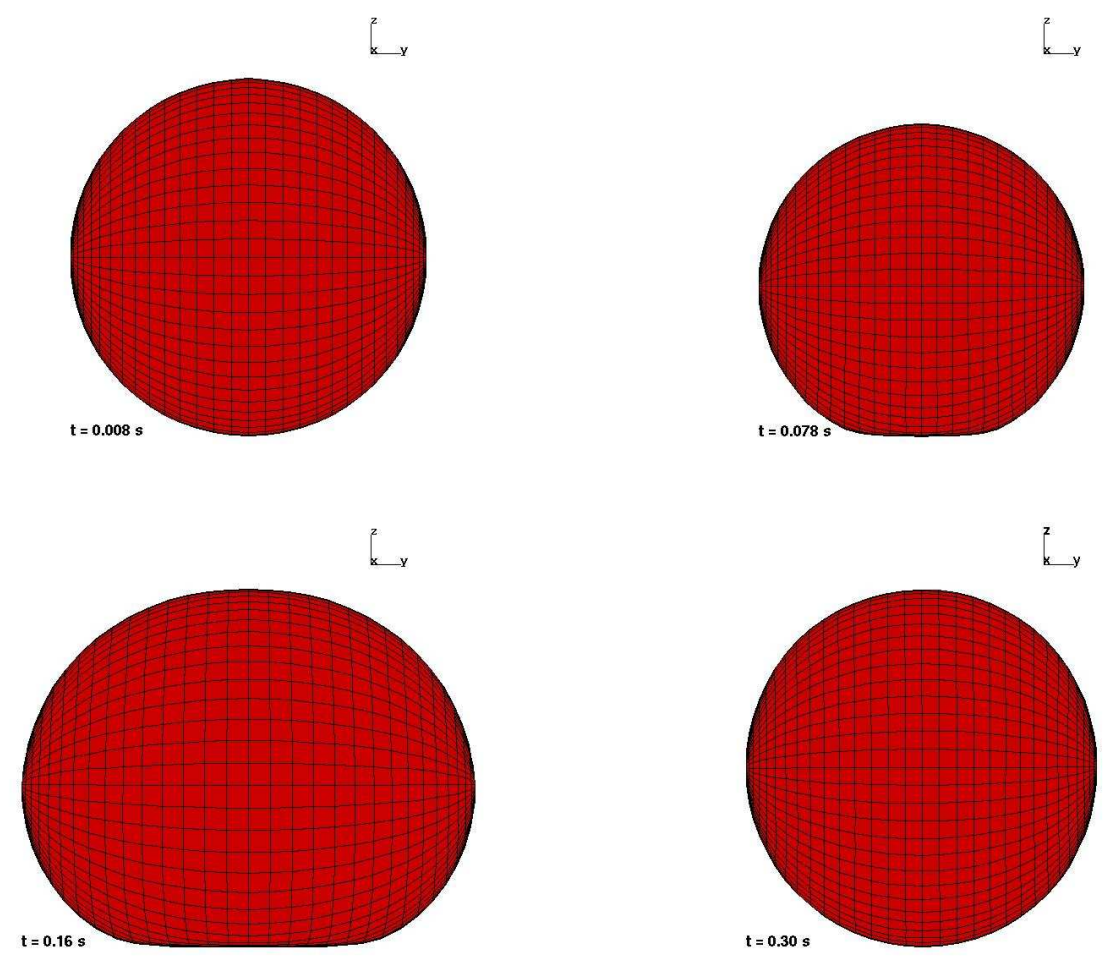

FIG. 5. Snapshots of the impact simulation. Images de la simulation du problème d'impact.

$$
\begin{aligned}
& m^{s}\left(\ddot{x}_{n+1 / 2}^{s}, \hat{U}^{s}\right)+\int_{x^{f}\left(\Omega_{0}^{f}, t_{n+1 / 2}\right)} \rho\left(\frac{U_{n+1}^{f}-U_{n}^{f}}{\Delta t}+\left[\left(U^{f}-U^{G}\right) \cdot \nabla U^{f}\right]_{n+1 / 2}\right) \cdot \hat{U}^{f} \\
& +\int_{\Omega_{0}^{s}} F_{n+1 / 2} \cdot\left(\Sigma_{n+1 / 2}^{c}+\Sigma_{n+1 / 2}^{i n c}\right): \nabla \hat{U}^{s} \\
& +\int_{\mathbf{x}^{f}\left(\Omega_{0}^{f}, t_{n+1 / 2}\right)}\left(\mu\left(\nabla_{x} U^{f}+\nabla_{x}^{t} U^{f}\right)-p I d\right)_{n+1 / 2}: \frac{\partial \hat{U}^{f}}{\partial x} \\
& =\int_{\Omega\left(t_{n+1 / 2}\right)} f_{n+1 / 2} \cdot \hat{U}+\int_{\partial \Omega\left(t_{n+1 / 2}\right)} g_{n+1 / 2} \cdot \hat{U}+\int_{\partial \Omega_{c}} \lambda_{n+1 / 2} \nu_{n+1 / 2} \cdot \hat{U}^{s} \\
& +\int_{\Gamma_{0}}\left(g_{\Gamma}\right)_{n+1 / 2} \cdot\left(\operatorname{tr}\left(\hat{U}^{s}\right)_{\mid \Gamma}-\operatorname{tr}\left(\hat{U}^{f}\right)_{\mid \Gamma}\right), \quad \forall\left(\hat{U}^{s}, \hat{U}^{f}\right) \in V^{s} \times V^{f},
\end{aligned}
$$

for momentum conservation, and

$$
\begin{aligned}
& \operatorname{tr}\left(\mathbf{x}_{n+1}^{s}\right)_{\mid \Gamma_{0}}=\operatorname{tr}\left(\mathbf{x}_{n+1}^{f}\right)_{\mid \Gamma_{0}}, \\
& \mathbf{x}_{n+1}^{f}=\operatorname{Ext}\left(\left(\mathbf{x}_{\mid \Gamma_{0}}^{s}\right)_{n+1}\right), U_{n+1 / 2}^{G}=\frac{\mathbf{x}_{n+1}^{f}-\mathbf{x}_{n}^{f}}{\Delta t},
\end{aligned}
$$

for the kinematic interface continuity and the fluid configuration map. 

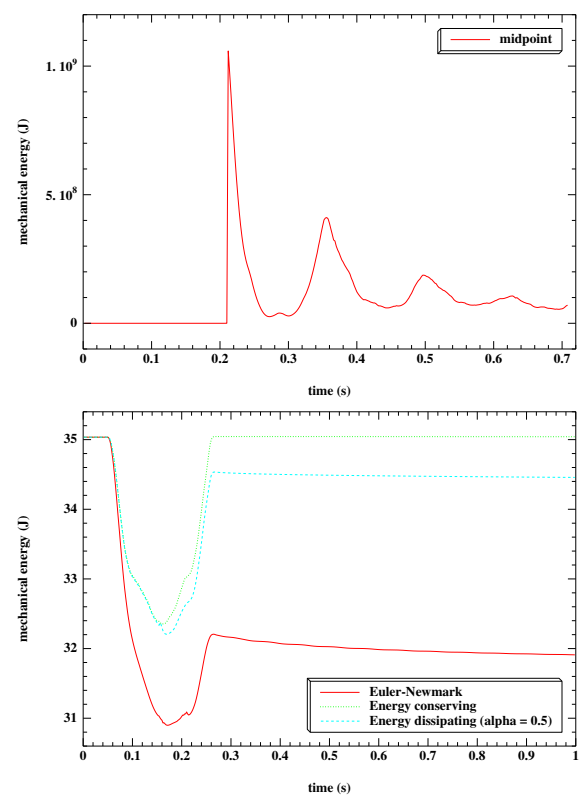

FIG. 6. Evolution of the ball mechanical energy through impact for midpoint, Euler-Newmark, energy conserving, and dissipating $(\alpha=0.5)$ schemes. Evolution de l'énergie mécanique de la balle pour les schémas de point milieu, d'Euler, conservatif et dissipatif $(\alpha=0.5)$.

\subsection{Energy balance for the fluid structure interaction problem}

A time integration of the principle of momentum conservation taking the real velocity field as test function indicates that the variation of the sum of the kinetic energy of the system and of the elastic energy of the structure must be equal to the difference between the energy introduced by the external boundary conditions and the energy dissipated by viscous effects inside the fluid. It is important to respect this energy principle after time discretization for stability purposes and for ensuring the long term accuracy of the numerical predictions.

To check energy conservation in the time discrete case, we need to multiply at each time $t^{n+1 / 2}$ the variational equation (11) by $U_{n+1 / 2}^{f}$ on the fluid, and by $U_{n+1 / 2}^{s}$ on the structure. This choice cancels the action of the interface traction forces $g_{\Gamma}$ because of the imposed kinematic compatibility condition (13) enforced at each time step $t_{n}$.

On the structure, we have seen above that the nonlinear energy corrections of Gonzalez [7] guarantee the exact conservation of energy, namely that the integration of the inertia terms directly yields the variation of the structural kinetic energy and that the integration of the stiffness terms directly produce the variation of elastic energy.

On the fluid, from the volume conservation equation (10) $\left(\operatorname{div}_{x}\left(U_{n+1 / 2}^{f}\right)=0\right.$ on $\left.x^{f}\left(\Omega_{0}^{f}, t_{n+1 / 2}\right)\right)$, a direct integration of the viscous and hydrostatic stresses directly yieds the viscous dissipation

$$
\int_{\mathbf{x}^{f}\left(\Omega_{0}^{f}, t_{n+1 / 2}\right)}\left(\mu\left(\nabla_{x} U^{f}+\nabla_{x}^{t} U^{f}\right)-p I d\right)_{n+1 / 2}: \frac{\partial U_{n+1 / 2}^{f}}{\partial x}=\int_{\mathbf{x}^{f}\left(\Omega_{0}^{f}, t_{n+1 / 2}\right)} 2 \mu\left|\frac{1}{2}\left(\nabla_{x} U_{n+1 / 2}^{f}+\nabla_{x}^{t} U_{n+1 / 2}^{f}\right)\right|^{2} .
$$

And finally, a direct integration of the inertia terms inside the fluid yields 


$$
\begin{aligned}
& I_{n+1 / 2}^{f}:=\int_{x^{f}\left(\Omega_{0}^{f}, t_{n+1 / 2}\right)} \rho \frac{U_{n+1}^{f}-U_{n}^{f}}{\Delta t} \cdot U_{n+1 / 2}^{f} \\
& +\int_{\mathbf{x}^{f}\left(\Omega_{0}, t_{n+1 / 2}\right)}\left[\rho\left(U^{f}-U^{G}\right)_{n+1 / 2} \nabla U_{n+1 / 2}^{f}\right] \cdot U_{n+1 / 2}^{f} \\
& =\int_{x^{f}\left(\Omega_{0}^{f}, t_{n+1 / 2}\right)} \frac{1}{2 \Delta t} \rho\left(\left|U_{n+1}^{f}\right|^{2}-\left|U_{n}^{f}\right|^{2}\right) \\
& +\int_{\mathbf{x}^{f}\left(\Omega_{0}, t_{n+1 / 2}\right)}-\frac{1}{2} \rho\left|U_{n+1 / 2}^{f}\right|^{2} \operatorname{div}\left[\left(U^{f}-U^{G}\right)\right]_{n+1 / 2} \\
& +\int_{\mathbf{x}^{f}\left(\Omega_{0}, t_{n+1 / 2}\right)} \operatorname{div} x\left[\frac{1}{2} \rho\left|U_{n+1 / 2}^{f}\right|^{2}\left(U^{f}-U^{G}\right)_{n+1 / 2}\right] .
\end{aligned}
$$

The last term disappears since we have $\left(U^{f}-U^{G}\right)_{n+1 / 2}=0$ on the interface from the kinematic condition (12) and the definition of $U_{n+1 / 2}^{G}$. After direct algebraic manipulations and substracting the weak equation of mass and the grid evolution law $\frac{1}{J} \frac{\partial J}{\partial t}=\operatorname{div}_{x}\left(U^{G}\right)$, we can reduce the inertia terms integral to

$$
\begin{aligned}
& I_{n+1 / 2}^{f}=\int_{\Omega_{0}^{f}} \frac{1}{2 \Delta t} \rho\left(J_{n+1}\left|U_{n+1}^{f}\right|^{2}-J_{n}\left|U_{n}^{f}\right|^{2}\right) \\
& \quad-\int_{\Omega_{0}^{f}} \frac{1}{8} \rho \frac{\partial J}{\partial t}\left|U_{n+1}^{f}-U_{n}^{f}\right|^{2}+\frac{\Delta t}{16} \rho \frac{\partial^{2} J}{\partial t^{2}}\left(\left|U_{n+1}^{f}\right|^{2}-\left|U_{n}^{f}\right|^{2}\right) \\
& \quad-\int_{\mathbf{x}^{f}\left(\Omega_{0}, t_{n+1 / 2}\right)}\left(\frac{1}{2}\left|U_{n+1 / 2}^{f}\right|^{2}-\hat{q}\right)\left(\operatorname{div}_{x}\left[\rho U^{f}\right]_{n+1 / 2}\right), \forall \hat{q} .
\end{aligned}
$$

The first line is the expected variation of kinetic energy. The second and third lines correspond to two types of discretization errors induced by the grid motion. The second line is proportional to the truncation error induced by the time discretization scheme of the Jacobian $J$, and directly depends on the regularity in time of the map $\rho J$. In other words, any abrupt changes of $J$ in time can lead to large local errors. The last line corresponds to a space truncation error

$$
e_{h}=\inf _{q_{h} \in Q_{h}} \int_{\Omega^{f}\left(t_{n+1 / 2}\right)} \frac{\Delta t}{2}\left(\frac{1}{2}\left|U_{n+1 / 2}^{f}\right|^{2}-q_{h}\right) \operatorname{div}\left[\rho U^{f}\right]_{n+1 / 2}
$$

which can be made very small by a careful choice of the space of pressure test functions $Q_{h}$. This error disappears for the space continuous problem.

These two second order errors are usually acceptable in most practical applications, because of the presence of viscous dissipation inside the fluid. In any case, these errors can be totally suppressed by introducing a new specific nonlinear second order correction in the fluid convection terms by setting

$$
\begin{aligned}
{\left[\left(U^{f}-U^{G}\right) \cdot \nabla U^{f}\right]_{n+1 / 2}=} & \left(U^{f}-U^{G}\right)_{n+1 / 2} \cdot \nabla\left(U^{f}\right)_{n+1 / 2}+\frac{1}{2} \operatorname{div}_{x}\left[U^{f}\right]_{n+1 / 2} U_{n+1 / 2}^{f} \\
& +\frac{1}{2}\left(-\operatorname{div}_{x}\left[U^{G}\right]_{n+1 / 2}+\frac{J_{n+1}-J_{n}}{\Delta t J_{n+1 / 2}}\right) U_{n+1 / 2}^{f} \\
& +\frac{J_{n+1}-J_{n+1 / 2}}{2 \Delta t J_{n+1 / 2}} \frac{\left|U_{n+1}^{f}\right|^{2}-\left|U_{n+1 / 2}^{f}\right|^{2}}{\left|U_{n+1 / 2}^{f}\right|^{2}} U_{n+1 / 2}^{f} \\
& +\frac{J_{n}-J_{n+1 / 2}}{2 \Delta t J_{n+1 / 2}} \frac{\left|U_{n+1 / 2}^{f}\right|^{2}-\left|U_{n}^{f}\right|^{2}}{\left|U_{n+1 / 2}^{f}\right|^{2}} U_{n+1 / 2}^{f} .
\end{aligned}
$$


Because of the continuous mass conservation equation, and of the grid evolution law $\frac{1}{J} \frac{\partial J}{\partial t}=\operatorname{div}_{x}\left(U^{G}\right)$, the additional terms are second order corrections. But they restore energy conservation, since we can now easily check the identity

$$
\begin{aligned}
& I_{n+1 / 2}^{f}:=\int_{x^{f}\left(\Omega_{0}^{f}, t_{n+1 / 2}\right)} \rho \frac{U_{n+1}^{f}-U_{n}^{f}}{\Delta t}+\left[\rho\left(U^{f}-U^{G}\right) \cdot \nabla U^{f}\right]_{n+1 / 2} \cdot U_{n+1 / 2}^{f} \\
& =\int_{x^{f}\left(\Omega_{0}^{f}, t_{n+1 / 2}\right)} \frac{1}{2 \Delta t J_{n+1 / 2}} \rho\left(J_{n+1}\left|U_{n+1}^{f}\right|^{2}-J_{n}\left|U_{n}^{f}\right|^{2}\right) .
\end{aligned}
$$

Remark 1 In [15], the use of the variable $\sqrt{J \rho} U^{f}$ was advocated as a possible way of preserving energy conservation within the fluid. But in practice, such a choice complexifies the calculation of the viscous term.

Remark 2 All nonlinear energy correction terms introduced above are second order. In a Newton's solution of the resulting problem, they can be omitted from the tangent stiffness matrix. In other words, they will be only added in the residuals, and never in the preconditioners.

\section{Multidomain solver}

After space and time discretization, we are faced at each time step with the numerical solution of a large scale coupled problem whose abstract form writes formally

$$
\begin{aligned}
& \left(\frac{\mathbf{M}^{s}}{\Delta t^{2}}+\mathbf{K}^{s}\right) X^{s}+B_{\Gamma s}^{t} G_{\Gamma}=F^{s}, \\
& \quad(\text { structure) } \\
& \left(\frac{\mathbf{M}^{f}}{\Delta t^{2}}+\mathbf{K}^{f}\right) X^{f}-B_{\Gamma f}^{t} G_{\Gamma}=F^{f}, \\
& \text { (fluid) } \\
& B_{\Gamma s} X^{s}-B_{\Gamma f} X^{f}=0, \quad \text { (interface matching). }
\end{aligned}
$$

This problem involves two nonlinear operators $\mathbf{K}^{s}$ and $\mathbf{M}^{f}$, for structural stiffness and fluid convection respectively. For the sake of simplicity, the fluid problem has been written in terms of displacements (instead of velocity), and the possible incompressibility constraints have been hidden. Traditionnally, such a coupled problem is solved by elimination of the fluid displacement $X^{f}$ and interface forces $G_{\Gamma}$, through the solution of a Dirichlet problem expressing them as a nonlinear function of the interface displacement $B_{\Gamma_{s}} X^{s}$ of the structure

$$
\left(\begin{array}{c}
X^{f} \\
G_{\Gamma}
\end{array}\right)=\left(\begin{array}{cc}
\frac{\mathbf{M}_{f}}{\Delta t^{2}}+\mathbf{K}^{f} & -B_{\Gamma_{f}}^{t} \\
-B_{\Gamma_{f}} & 0
\end{array}\right)^{-1}\left(\begin{array}{c}
F^{f} \\
-B_{\Gamma_{s}} X^{s}
\end{array}\right) .
$$

This formally reduces the coupled problem to a single structural problem with added terms in the mass and stiffness operator coming from the elimination of the fluid unknowns

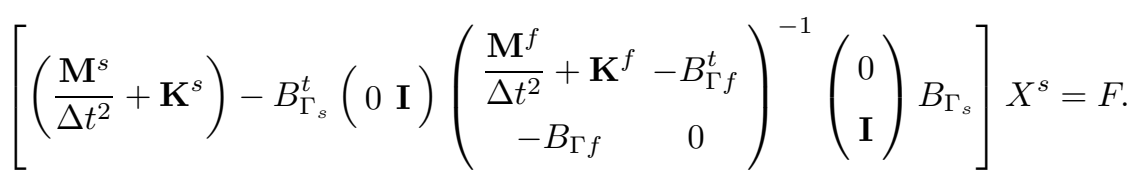


But, in complex three dimensional situations, unless using a very small modal basis for describing the structural motion, the direct solution of the above system is untractable. Domain decomposition techniques give simple ways of solving it as a succession of local problems. In a Dirichlet Neumann algorithm, the system (15) in $X^{s}$ is solved by an iterative algorithm using the structural matrix $\left(\frac{\mathbf{M}^{s}}{\Delta t^{2}}+\mathbf{K}^{s}\right)$ as a preconditioner, therefore dropping the added mass and stiffness contributions of the fluid to the structure during the preconditioning step. The corresponding algorithm takes the simple form

$$
\begin{aligned}
& X^{s}=X^{s}-\rho\left(\frac{\mathbf{M}^{s}}{\Delta t^{2}}+\mathbf{K}^{s}\right)^{-1} R \\
& =X^{s}-\rho\left[X^{s}-\left(\frac{\mathbf{M}^{s}}{\Delta t^{2}}+\mathbf{K}^{s}\right)^{-1}\left\{B_{\Gamma_{s}}^{t}\left[\left(\begin{array}{ll}
0 & \mathbf{I}
\end{array}\right) \mathbf{D}^{f^{-1}}\left(\begin{array}{l}
0 \\
\mathbf{I}
\end{array}\right)\right] B_{\Gamma_{s}} X^{s}-F\right\}\right] .
\end{aligned}
$$

In each iteration, a Dirichlet problem with operator $\mathbf{D}^{f}=\left(\begin{array}{cc}\frac{\mathbf{M}^{f}}{\Delta t^{2}}+\mathbf{K}^{f}-B_{\Gamma f}^{t} \\ -B_{\Gamma f} & 0\end{array}\right)$ must first be inverted on the fluid in order to compute the residual

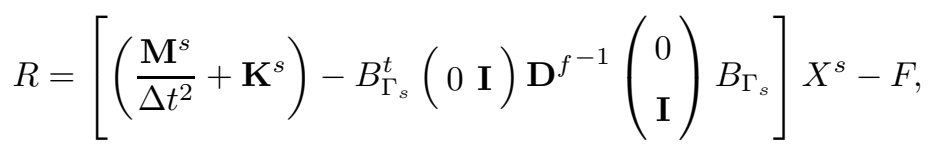

and a Neumann problem is then solved in the structure to compute the solution update $\left(\frac{\mathbf{M}^{s}}{\Delta t^{2}}+\mathbf{K}^{s}\right)^{-1} R$, hence the name Dirichlet Neumann given to this type of algorithm.

This in fact reduces the original coupled problem to a fixed point formulation written with respect to the interface displacement $B_{\Gamma_{s}} X^{s}$

$$
B_{\Gamma_{s}} X^{s}=\left[B_{\Gamma_{s}}\left(\frac{\mathbf{M}^{s}}{\Delta t^{2}}+\mathbf{K}^{s}\right)^{-1} B_{\Gamma_{s}}^{t}\right]\left[\left(\begin{array}{ll}
0 & \mathbf{I}
\end{array}\right) \mathbf{D}^{f^{-1}}\left(\begin{array}{l}
0 \\
\mathbf{I}
\end{array}\right)\right] B_{\Gamma_{s}} X^{s}+R
$$

It was proved in [17] that the Dirichlet Neumann operator

$$
\left[B_{\Gamma_{s}}\left(\frac{\mathbf{M}^{s}}{\Delta t^{2}}+\mathbf{K}^{s}\right)^{-1} B_{\Gamma_{s}}^{t}\right]\left[\left(\begin{array}{ll}
0 & \mathbf{I}
\end{array}\right) \mathbf{D}^{f^{-1}}\left(\begin{array}{l}
0 \\
\mathbf{I}
\end{array}\right)\right]
$$

appearing in this fixed point problem is bounded (at least in a linear framework), ensuring the convergence of an accelerated fixed point algorithm, if $\rho$ is properly chosen in (16). This theoretical analysis also shows the key importance of a correct treatment of the added mass terms for stability. These terms express that any acceleration of the structure implies a motion on its interface, and is slowed down by the incompressiblity condition inside the fluid which generates a pressure field in opposition to this motion.

The problem encountered in many numerical experiments is to properly choose the coefficient $\rho$, an improper choice leading to a large number of fixed point iterations (16) at the corresponding time step (typically fifty or more). This difficulty was finally overcome in [6] who have proposed to solve the Dirichlet Neumann problem (17) not by an accelerated fixed point iteration, but by a quasi Newton algorithm, the inversion of the tangent matrix being replaced by the solution of a simplified linear fluid structure problem obtained by replacing the real fluid by a perfect incompressible fluid, and by linearizing the structural stiffness. The simplified fluid structure problem respects the basic coupling mechanism between the fluid and the structure, in particular the added mass effect, and is easy to solve. Its solution is usually 
obtained after a small number of GMRES iterations [13], solving successively a Poisson equation for the fluid pressure and a linear structural problem with known and factored stiffness matrix. In practice, the global solution of (17) at each time step only requires of the order of 10 Quasi Newton iterations, each quasi Newton iteration involving an average of 8 GMRES iterations. The major cost in this algorithm is to compute the residual of the nonlinear Dirichlet Neumann problem (17), and is therefore directly proportional to the number of quasi Newton iterations, and rather insensitive to the number of linear GMRES iterations. Such a performance is illustrated below on the numerical solution of a blood flow inside an anevrism.

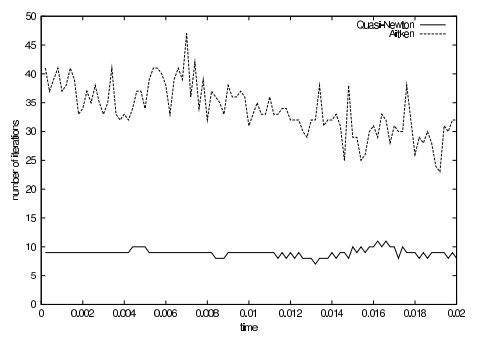

FIG. 7. Quasi Newton compared to accelerated fixed point iterations. Nombre d'itérations comparé entre Quasi-Newton et point fixe.

\section{Conclusion}

Energy conserving nonlinear time integration schemes have been introduced, described and justified for fluid structure problems. We have generalized this strategy to all components of the system : compressible

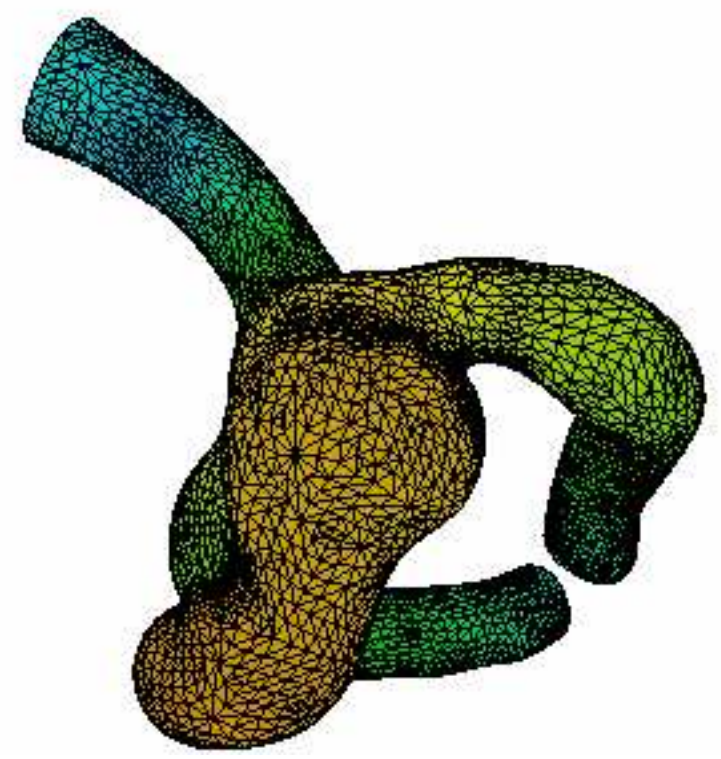

FIG. 8. A pressure wave inside a complex anevrism. Onde de pression à l'intérieur d'un anévrisme. 
stiffness, incompressibility constraint, frictionless contact, fluid convection in a moving grid. We have also reviewed a recent extension of Dirichlet Neumann algorithms which reduces the solution of the global coupled problem to a sequence of structural and fluid poblems. Different numerical simulations on challenging three dimensional problems have illustrated the numerical efficiency of these procedures.

In the context of biomechanics, the problem is now to develop better structural models for handling membrane locking phenomena for general grids as obtained from medical imaging, to upgrade the physiological model of the structures, to get a better insight on the adequate boundary conditions, and to develop proper identification strategies based on medical imaging to upgrade the predictability of the models.

\section{Références}

[1] F. Armero and E. Petcz. Formulation and analysis of conserving algorithms for frictionless dynamic contact/impact problems. Comp. Meth. in Appl. Mech. and Eng., (158) :269-300, 1998.

[2] K-J. Bathe. Finite Element Procedures in Engineering Analysis. Prentice-Hall, 1982.

[3] M.A. Crisfield. Nonlinear Finite Element Analysis of Solids and Structures, volume 2 : Advanced Topics. Wiley, 1997.

[4] R. Dautray and J.L. Lions. Mathematical Analysis and Numerical Methods for Science and Technology. Springer Verlag, Berlin, 1990.

[5] E. de Langre. Fluides et Solides. Editions de l'Ecole Polytechnique, Palaiseau, 2001.

[6] J.F. Gerbeau and M. Vidrascu. A quasi-newton algorithm based on a reduced model for fluid-structure interaction problems in blood flows. M2AN, 37 :663-680, 2003.

[7] O. Gonzalez. Exact energy and momentum conserving algorithms for general models in nonlinear elasticity. Comp. Meth. in Appl. Mech. and Eng., 190(13-14) :1763-1783, December 2000.

[8] P. Hauret. Méthodes numériques pour la dynamique des structures nonlinéaires incompressibles à deux échelles. $\mathrm{PhD}$ thesis, Ecole Polytechnique, 2004.

[9] T.A. Laursen and V. Chawla. Design of energy conserving algorithms for frictionless dynamic contact problems. Int. J. Num. Meth. Engr., 40 :863-886, 1997.

[10] T.A. Laursen and G.R. Love. Improved implicit integrators for transient impact problems; geometric admissibility within the conserving framework. Int. J. Num. Meth. Engr., 53(2) :245-274, January 2002.

[11] S. Mani. Truncation error and energy conservation for fluid structure interactions. Comp. Meth. in Appl. Mech. and Eng., 192(43) :4769-4804, 2003.

[12] A. Quarteroni and A. Valli. Domain Decomposition Methods for Partial Differential Equations. Oxford University Press, 1999.

[13] Y. Saad. Iterative Methods for Sparse Linear Systems. PWS, 1996.

[14] J.C. Simo and N. Tarnow. The discrete energy-momentum method. conserving algorithms for non linear elastodynamics. $Z$ angew Math Phys, 43 :757-792, 1992.

[15] P. Le Tallec and P. Hauret. Energy conservation in fluid-structure interactions. In P. Neittanmaki Y. Kuznetsov and O. Pironneau, editors, Numerical Methods for Scientific Computing, Variational Problems and Applications, pages 94-107, CIMNE, Barcelona, 2003.

[16] P. Le Tallec and S. Mani. Conservation laws for fluid structure interactions. In T. Kvamsdal, editor, International Symposium on Computational Methods for Fluid Structure Interactions, pages 61-78, Trondheim, 1999. Tapir.

[17] P. Le Tallec and J. Mouro. Fluid structure interaction with large structural displacements. Comp. Meth. in Appl. Mech. and Eng., 190(24-25) :3039-3068, 2001. 